\title{
The Function of tPA in Fibrinolysis is Analogous to the Starter of An Automobile
}

\author{
Victor Gurewich* \\ Department of Medicine, Harvard Medical School, Mount Auburn Hospital, USA
}

*Corresponding author: Victor Gurewich, Department of Medicine, Harvard Medical School, Mount Auburn Hospital, USA.
Received Date: April 16, 2021

Published Date: May 11, 2021

\section{tPA Versus Streptokinase}

Therapeutic fibrinolysis has used tissue plasminogen activator (tPA) as the fibrinolytic of choice ever since tPA was given FDA approval for the treatment of acute myocardial infarction (AMI) in 1987. This was followed in few years by its approval for ischemic stroke and tPA re-mains the treatment today based on an assumption that tPA is responsible for biological fibrinolysis. However, the evidence for this was never strong but has remained unchallenged for the past 33 years. When tPA became the fibrinolytic of choice it was on the results of three clinical trials in almost 100,000 patients with AMI in whom treatment with tPA was compared with streptokinase (SK), the fibrinolytic at the time. However, SK is an indirect activator that has first to form a complex with plasminogen or plasmin before it becomes an activator, which means that SK consumes its own substrate and the fibrinolytic itself, an inefficient mechanism of action. By contrast, tPA is a direct plasminogen activator. Nevertheless, in the first two trials the mortalities with tPA and SK were identical. Finally, in a third trial, which was divided into four groups, a significantly lower mortality was found but only in one of the four groups [1-3]. In addition, there were significantly more hemorrhagic side effects with tPA than SK.

A subsequent Bayesian analysis of these results concluded that the efficacy of tPA over SK had not been established [4]. Nevertheless, tPA received FDA approval for the treatment of AMI, and a few years later it was also approved for the treatment of ischemic stroke. This was the evidence on which the use tPA was based and the question of whether tPA is really more effective than SK was never readdressed to my knowledge. Instead, alternatives to fibrinolysis are now being adopted which are mechanical interventions like percutaneous coronary intervention (PCI), that has become the treatment of choice for AMI, or thrombectomy which is being used in large vessel strokes. Since these are time consuming procedures, they are poorly adapted to salvage the function of an ischemic myocardium or brain. Fibrinolysis remains the simplest and fastest method to reestablish blood flow and therefore the most promising one. However, a more effective and safer plasminogen activator than tPA monotherapy is required.

\section{Biological Fibrinolysis Provides A Better Model for Therapeutic Fibrinolysis}

In addition to tPA, there is a second plasminogen activator present in normal blood called urokinase plasminogen activator (uPA). The native form of uPA is a single chain proenzyme (prouPA) discovered in 1980 [5], the precursor of its two-chain enzymatic form (tcuPA), called urokinase, had been known for years. Unlike tcuPA, prouPA is a fibrin-specific fibrinolytic like tPA, but unlike tPA it does not bind to fibrin and is a proenzyme. Its fibrinolytic properties are complementary to those of tPA, which makes their combined fibrinolytic effect synergistic [6]. Their effects are also sequential with tPA initiating fibrinolysis when it binds to fibrin at its one-binding site on the fibrin D-domain. This enables tPA to activate the single fibrin bound plasmin-ogen on intact fibrin, thereby initiating fibrinolysis.

Fibrin degradation then creates two additional plasminogen binding sites which are on the fibrin E-domain. The first of these undergoes an unusual conformational change that has three C-terminal lysins. This conformation enables prouPA to activate it and plasmin then activates prouPA to tcuPA, and this activates the 
final fibrin-bound plasminogen [7]. A sequential combination of a small dose tPA followed by prouPA induced more effective lysis with smaller doses than tPA alone [8]. This combination was once tested in 101 patients with AMI. The patients were treated with a $5 \mathrm{mg}$ bolus of tPA followed by a 90 minute infusion of prouPA at $40 \mathrm{mg} / \mathrm{h}$. This induced an $82 \%$ TIMI-3 coronary artery patency at $24 \mathrm{~h}$ and a $1 \%$ mortality in patients. This compared favorably with the largest tPA monotherapy study (GUSTO) which required $100 \mathrm{mg} / \mathrm{h}$ of tPA and induced a TIMI- 3 patency in $45 \%$ of the patients at $24 \mathrm{~h}$ and a mortality in $6 \%$ at one month [9]. Had this sequential combination replaced tPA monotherapy at the time it was published, almost one million AMI deaths in the US alone could have been averted.

\section{Conclusion}

Monotherapy with tPA has been used for therapeutic thrombolysis for the past 33 years and requires $100 \mathrm{mg}$ of tPA infused over 60 minutes. By contrast, in biological fibrinolysis tPA is limited to the initiation of fibrinolysis and which requires only a $5 \mathrm{mg}$ bolus. Thereafter, lysis is continued by uPA (prouPA and tcuPA). There is only one plasminogen binding site located on the fibrin D-domain near that of tPA which enables it to be activated by tPA with synergistic efficiency. Lysis creates two new plasminogen binding sites and their plasminogen's are activated by prouPA and tcuPA rather than tPA. A $5 \mathrm{mg}$ tPA bolus followed by a $40 \mathrm{mg} / \mathrm{h}$ prouPA infusion was once used to treat 101 AMI patients. This combination almost doubled the TIMI-3 coronary patency and reduced mortality 6-fold compared to tPA monotherapy. By using the natural biological model of fibrinolysis in place of tPA monotherapy both the safety and efficacy of therapeutic thrombolysis could be much improved.

\section{Acknowledgement}

None.

\section{Conflict of Interest}

No conflict of interest.

\section{References}

1. (1990) GISSI-2: A factorial randomized trial of alteplase versus streptokinase and heparin versus no heparin among 12,490 patients with acute myocardial infarction. Lancet 336: 65-71.

2. (1992) ISIS-3: A randomized compari-son of streptokinase vs streptokinase plus aspirin plus heparin vs aspirin alone among 41,299 cases of suspected myocardial infarction. Lancet 339: 65-71.

3. The GUSTO Investigators (1993) An international randomized trial comparing four thrombolytic strategies for acute myocardial infarction. N Eng J Med 329: 673-682.

4. Brophy JM, Joseph L (1995) Placing trials in context using Bayesian analysis. GUSTO revisited by Reverend Bayes. JAMA 273: 871-875.

5. Husain SS, Gurewich V, B Lipinski (1983) Purification and partial characterization of a single chain, high molecu-lar weight form of urokinase from human urine. Arch Biochem Biophy 220(1): 31-38.

6. Pannell R, Black J, Gurewich V (1988) The complementary modes of action of tissue plasminogen ac-tivator (t-PA) and pro-urokinase (proUK) by which their synergistic effect on clot lysis may be explained. J Clin Invest 81: 853-859.

7. Gurewich V (1989) The sequential, complementary, and synergistic activation of fibrin-bound plasminogen by tissue plasminogen activator and pro-urokinase. Fibrinolysis 3: 59-66.

8. Gurewich V (2013) Fibrinolytic mechanisms of tPA, prouPA, mutant prouPA and their implications for therapeutic thrombolysis. Cardiovasc Eng Technol 4(4): 328-338.

9. Pannell R, Li S, Gurewich V (2015) Highly effective fibrinolysis by a sequential synergistic combina-tion of mini-dose tPA plus low-dose mutant proUK. PLOS One 10: e0122018. 